Dominancia

Cerebral y

Educación

Universitaria

Marta R. Mendieta Alonso

Cesar A. Briones Mendieta 


\section{Dominancia \\ Cerebral y \\ Educación \\ Universitaria}

\section{Cerebral Dominance and University education}

\section{Marta R. Mendieta Alonso \\ Docente-Investigadora \\ Departamento de Psicología \\ Fac. de Humanidades y Ciencias Jurídicas \\ UNAN-Managua \\ ORCID: 000-0001-6890-4354 \\ mmendieta@unan.edu.ni}

\section{César A. Briones Mendieta}

Doctorante y asistente de investigación Departamento de Enseñanza, Aprendizaje y Estudios Educativos (TLES)

Western Michigan University-USA

ORCID: 0000-0002-4176-4889

cesar.briones@wmich.edu

(c) (i)(2) Copyright 02017 UNAN-Manag

\section{RESUMEN}

Los descubrimientos en neurociencia sobre dominancia cerebral y sus múltiples aplicaciones para la vida han ido en aumento. Hay muchos trabajos investigativos de aplicación de la neurociencia en la mejora educativa, aunque existen pocos a nivel de educación superior. Este estudio tuvo dos propósitos; el primero, identificar y comparar los perfiles de dominancia cerebral de los estudiantes de la carrera de Psicología de la UNAN-Managua en distintos años académicos (2009, 2010 y 2013). El segundo, verificar la dominancia cerebral en una muestra de estudiantes, al inicio y al final de su carrera. Los resultados de la investigación evidencian una dominancia simple, la preferencia del hemisferio izquierdo, sobre todo del Cortical Izquierdo; Baja dominancia en el hemisferio derecho en especial del cuadrante Límbico Derecho. Por otro lado, con base en la comparación de los puntajes obtenidos en el pre test y post test, se observa que no hay cambios con respecto a la dominancia de los diferentes cuadrantes tanto del hemisferio Izquierdo como del Derecho. Para evaluar la tendencia a utilizar los cuatros cuadrantes por los estudiantes, de acuerdo con el modelo de cerebro total propuesto por Ned Herrmann (1989), se utilizó el test de Chalvin (1993), instrumento auto administrado que se responde en un lapso de 15 a 20 minutos y consta de 22 ítems (con cuatro opciones de respuesta, de las cuales se eligen una o dos) relacionados con actividades de estudio y de la vida diaria. Las respuestas se trasladan a una plantilla de corrección y, sumadas las puntuaciones por cuadrante, se obtiene el perfil.

\section{PALABRAS CLAVE}

\section{Dominancia cerebral, educación superior}

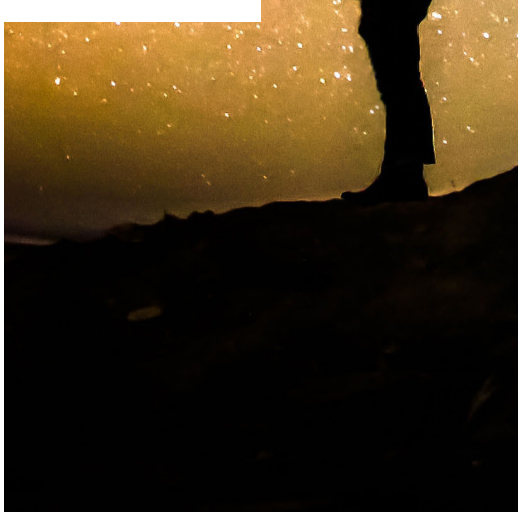




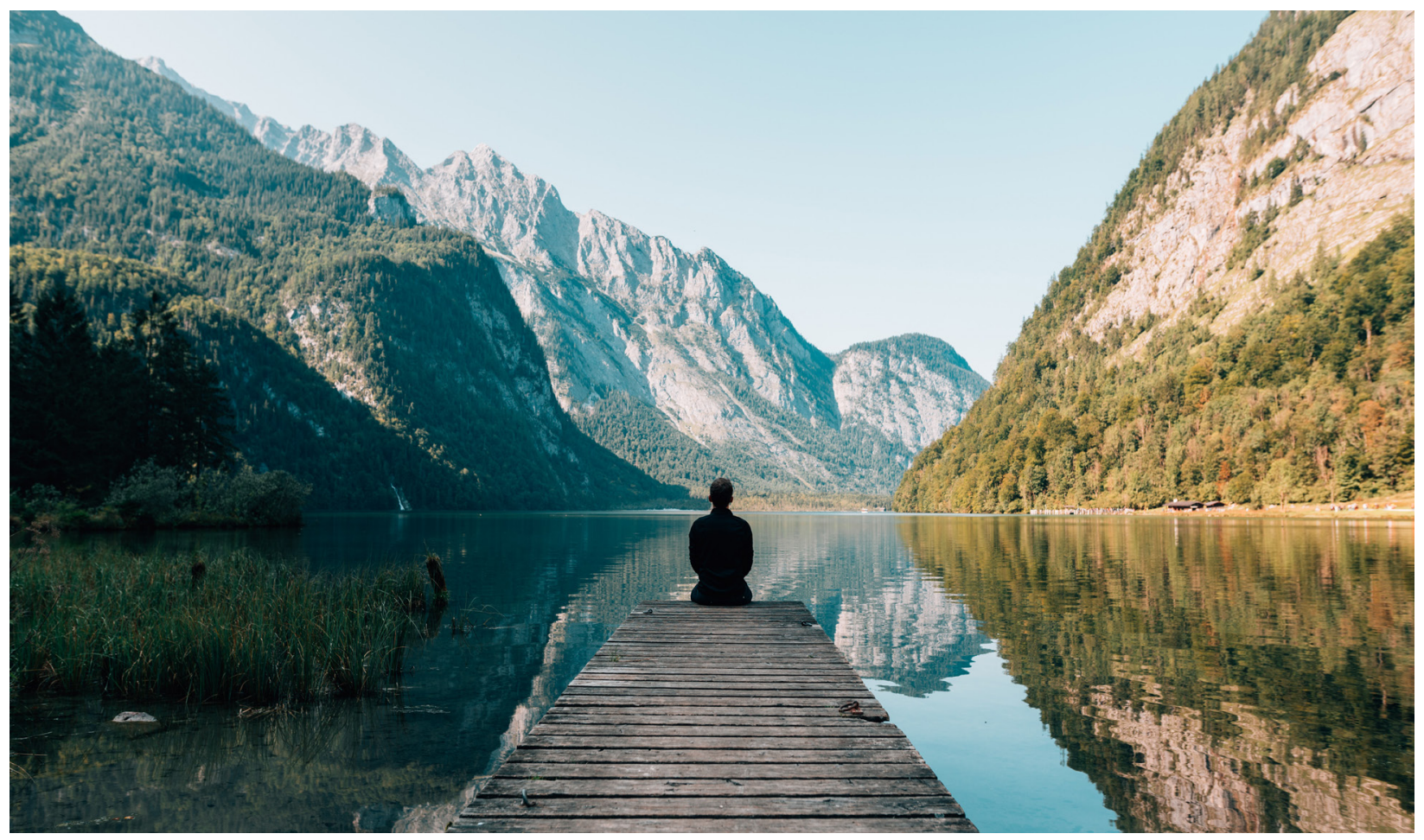

\section{ABSTRACT}

The discoveries neuroscience about cerebral dominance and its multiple applications for life have been increasing. There are many research works on the application of neuroscience in educational improvement, although there are few at the higher education level. This work had two purposes; the first one was identify and compare the cerebral dominance profile of phycology students of UNAN-Managua from different academic years (2009, $2010,2013)$. The second one, was verify the cerebral dominance in a sample of students at the beginning and end of their undergraduate studies. The investigation results show a simple dominance, the preference of Left Brain Hemisphere particularly of Left cortical; Lower Dominance on Right Brain Hemisphere specially of Right Limbic quadrant. On the other side, based on the comparison of scores obtained from the pre and post test, it observes that there are no changes related to the dominance of the different quadrants of both the Left Brain Hemisphere and Left Brain Hemisphere. To evaluate the tendency to use the four quadrants by students, according to the Whole Brain Model by Ned Hermann (1989) the Chalvin test (1993) was used, a self-administrated instrument which is answered within 15 or 20 minutes, this consists of 22 items (with four response options, of which one or two are chosen) related to the study activities and daily life. The answers are transferred to a correction template and, added the scores per quadrant, the profile is obtained.

\section{KEYWORDS}

\section{Cerebral dominance, higher education.}




\title{
Introducción
}

E n los años 90, conocidos como la "década del cerebro", se realizaron múltiples investigaciones sobre el funcionamiento del cerebro y sus potencialidades, dando como resultado conocimientos más amplios y precisos. En este sentido, Herrmann (1989), quien realizó su modelo y estudios basados en los de Sperry, sobre la especialización de los hemisferios (1981) y los de MacLean, relativos a coexistencia de tres cerebros en uno Cerebro Triuno- (1962, citado en Chalvin, 2003), reconoce el modelo de Cerebro Total. Estos resultados han llevado a replantearse si la educación es suficiente, adecuada y óptima para desarrollar las múltiples y diferentes capacidades que potencialmente se encuentran en el cerebro o si, por el contrario, como bien señala Ornstein (1977) solamente se está adecuando la educación para la utilización de la mitad del cerebro.

Han pasado más de 40 años y en muchos países se han efectuado investigaciones en el ámbito educativo inspirado en el modelo de Cerebro Total de Herrmann para demostrar la importancia de la Dominancia Cerebral en la Educación. Muchos han llegado a la conclusión de que se está educando de forma parcial y deficiente, favoreciendo el desarrollo del hemisferio izquierdo en detrimento del hemisferio derecho (Ornstein, 1977; Gardie, 1997).

El acelerado desarrollo de las neurociencias permite mayor comprensión del funcionamiento del cerebro y debe, necesariamente, ser incorporado a los estudios sobre el mejoramiento de la habilidad del docente para enseñar y la del estudiante para aprender. Se trata de comprender el origen de las diferencias individuales en el comportamiento y aprendizaje de los estudiantes, y a la vez entender que la pedagogía contemporánea es un espacio de la mente y de los fenómenos mentales.

Como bien señala Barrios (2000, p. 4), "los avances de la neurociencia han ampliado los límites del espacio pedagógico, más allá de una simple expansión, pues habrá que no sólo definir los límites de ese espacio pedagógico sino los límites de la educación". El enfoque neuropsicológico y, más concretamente de hemisfericidad cerebral y el modelo de cerebro total de Herrmann en el campo educativo no se ha aprovechado lo suficiente. En las teorías de la educación, generalmente, no teorizan sobre el cerebro y los procesos cerebrales del estudiante; ni el sentido y orientación de la docencia. La nueva pedagogía está orientada hacia la innovación y la creatividad, nacida de la interconexión entre la pedagogía y las neurociencias. Demanda a la educación básica la estimulación y el aprendizaje temprano y a la educación superior que los estudiantes promuevan situaciones problematizadoras en su aprendizaje, de los diversos eventos de la naturaleza, sociedad o del hombre y que lo transforme en investigador de sus actos y su entorno: es decir crítico y creativo.

\author{
"los avances de la \\ neurociencia han ampliado \\ los limites del espacio \\ pedagógico...
}


En el congreso Hispanoamericano de Investigación Educativa en Caracas, en 1992, como parte de su conferencia sobre el Proceso Creador a la luz de la Neurociencia, el doctor Martínez concluye que nuestros sistemas educativos donde se cultiva de forma casi exclusiva el hemisferio izquierdo, sus funciones racionales conscientes, descuidando la intuición y las funciones holísticas y gestálticas del hemisferio derecho, marginando el componente emotivo y afectivo, era el responsable de la falta de fe en nosotros mismos, la falta de una escolarización apropiada, la falta de un desarrollo estructural oportuno en las etapas evolutivas de la persona con consecuencia funcionales catastróficas y de una evaluación escolar inhibidora que coarta potencialidades, iniciativas e impulsos del estudiante.

El modelo de Cerebro Total de Herrmann (1989) plantea la existencia de cuatro cuadrantes o estilos de pensamiento. Ellos constituyen cuatro modalidades autónomas de procesamiento diferencial de información, las cuales pueden ser convenientemente desplegadas de forma individual o combinadas, tanto secuencia como simultáneamente en los diferentes procesos de funcionamiento cerebral. Herrmann reformula el funcionamiento del cerebro integrado por la neo corteza (hemisferio derecho-izquierdo) con el sistema Límbico (cerebro medio) y concibe al cerebro integrado como una totalidad orgánica dividida en cuatro cuadrantes o áreas, todas ellas directa o indirectamente conectadas entre sí por el cuerpo calloso y otras comisuras. De esto se concluye que la capacidad total está desaprovechada.

Los cuadrantes, conocidos como Cortical Izquierdo (Cl), Límbico Izquierdo (LI), Límbico Derecho (LD) y Cortical Derecho (CD) corresponden a cuatro modos determinados, distintos e independientes de procesamiento diferencial de información, representan formas distintas en que la persona opera, piensa, crea, aprende y, en suma, convive con el mundo y consigo misma, aun cuando se reconoce que el cerebro funciona como una totalidad integrada.

Las características, procesos de pensamiento y competencias (Chalvin, 2003, p. 78) que posee cada uno de los cuadrantes cerebrales, según Ned Herrmann, son:

Cortical Izquierdo (Cl): sus características son frio, distante, pocos gestos, voz elaborada, intelectualmente brillante, capaz de evaluar y criticar, irónico, competitivo, individualista entre los procesos cognitivos que desarrolla destacan el análisis, razonamiento, lógica, rigor y claridad; tendencia por los modelos y teorías, colecciona hechos, procede de hipótesis, prefiere la palabra precisa y las competencias que posee son la abstracción y resolución de problemas.

Límbico Izquierdo (LI): sus características son introvertido, emotivo, controlado, minucioso, maniático, tiende a monologar, gusto por las fórmulas, conservador y fiel, defensa del territorio, vinculación a la experiencia y ama al poder, entre los procesos cognitivos que desarrolla se encuentran la planificación, formalización, estructura, definición de procedimientos, secuencial, verificador, ritualista y metódico. Las competencias que posee son la administración, el sentido de organización, la realización y puesta en marcha, conductor de hombres, orador y trabajador consagrado.

Límbico Derecho (LD): sus características son extrovertido, emotivo, espontáneo, gesticulador, lúdico, hablador, espiritual, aquiescente, reacciona mal antes las críticas, desarrolla la integración mediante la experiencia; se mueve por el principio del placer; fuerte implicación afectiva; trabaja con base en sentimientos; escucha y pregunta; siente la necesidad de compartir y de vivir en armonía; evalúa los comportamientos. Y las competencias que posee son la relacional, contacto humano, el diálogo, la enseñanza, trabajo en equipo, expresión oral y escrita.

Cortical Derecho (CD): tiene como características la originalidad, sentido del humor, gusto por el riesgo, espacialidad, gusto por las discusiones, futurista, discurso brillante, independencia. 
Los conceptos que desarrolla son la conceptualización, síntesis, imaginación, globalización, intuición, visualización, asociación, integración de imágenes y metáforas y las competencias que posee son; creación, espíritu empresarial, visión del futuro, artista e investigación.

Desde el punto de vista de su contextualización estructural y teniendo en cuenta que es un modelo metafórico, de acuerdo con Caza (2005), este se puede representar de la siguiente manera:

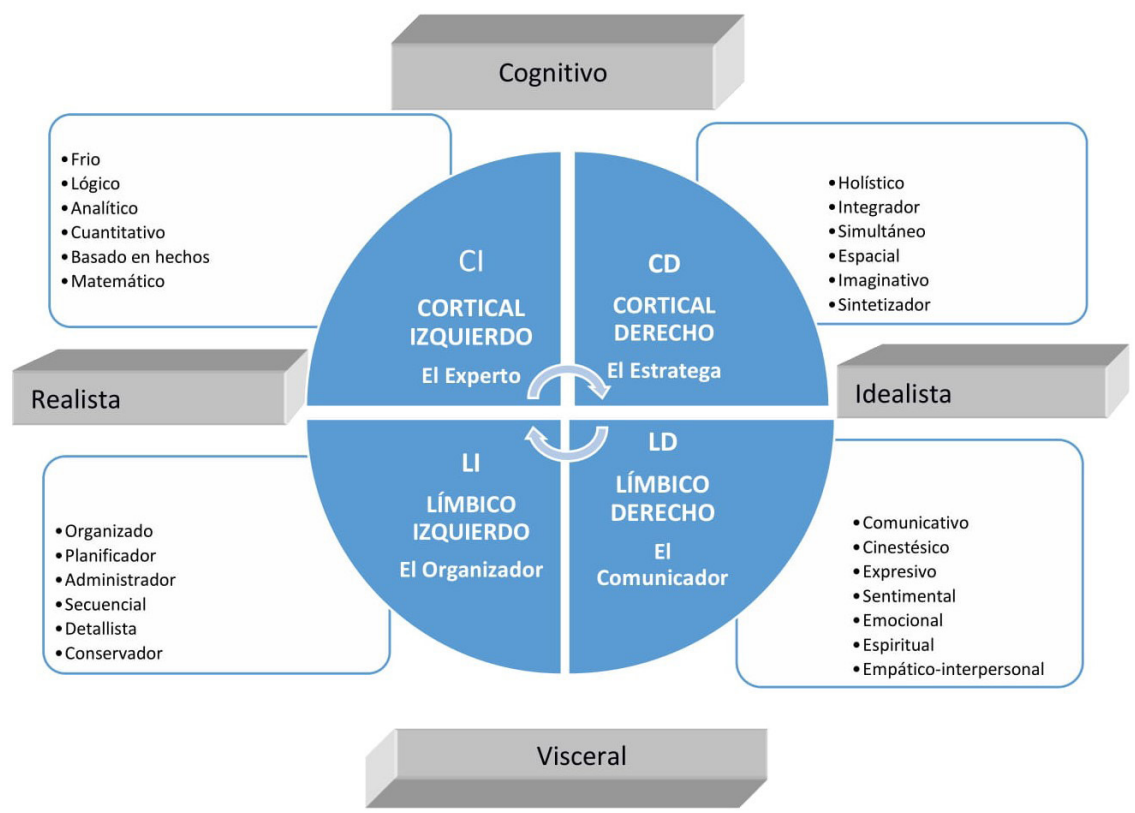

Fuente: Propia, tomado de Caza (2005)

De esta manera se reconocen las características, procesos y competencias que tienen las personas de acuerdo con su dominancia cerebral. Una persona que es predominantemente cortical (izquierdo-derecho) se considera cognitivo, los límbicos (izquierdo-derecho) se consideran personas viscerales; en los que predomina el hemisferio izquierdo (cortical - límbico) son realistas y en quienes predomina el hemisferio derecho (cortical-límbico) se considera que son personas idealistas.

Gardie (2000) a través de un estudio comparativo de perfil de estilos de pensamiento en una muestra de profesionales, distribuidos en nueve categorías y perfiles obtenidos en docentes en años anteriores (1997), señala que la naturaleza de un perfil determinado refleja la historia de un individuo y representa una orientación definida en cuanto a sus habilidades, destrezas, conocimientos y hábitos, creencias, valores, etc. De igual manera un grupo definido por su profesión- ocupación estará representado por un perfil distinto, según el área del conocimiento al que pertenece. Así, Gardie concluye que en la muestra de profesionales se obtuvieron perfiles similares a los reportados en la muestra de docentes en 1997, esto es, una preferencia límbica por encima de la cerebral y un predominio del hemisferio izquierdo sobre el derecho, lo cual explica con base en factores socio-históricos de la evolución de la sociedad venezolana e hipotetiza que podrían coincidir con la realidad de otras naciones de América Latina. 
Además, demuestra que el "sub aprovechamiento del potencial creativo, el insuficiente desarrollo de los múltiples talentos de la compleja estructura cerebral, así como el funcionamiento de los estilos de pensamiento es incompleto, insuficiente, escasamente desplegado y explotado" (Gardie 2000, p.15).

En el diagnóstico de estilos de aprendizaje en estudiantes universitarios de las distintas carreras de la Universidad Autónoma de San Luis Potosí (México), basados en la dominancia cerebral y realizada por Gómez Sánchez A, y Colab. (2009-2010), se obtuvo resultados similares; una alta preferencia por el cuadrante Cortical Izquierdo $(\mathrm{Cl})$, seguido por una preferencia media del límbico izquierdo (LI). No se encontraron evidencia de relación con variables sociodemográficas.

Las universidades están preocupadas y siguen esforzándose para que los currículos y programas educativos se dirijan a desarrollar el potencial del que es capaz el cerebro de los adolescentes y jóvenes que ingresan a ella. Las interrogantes que surgen ante estos esfuerzos convienen en ¿qué tanto están dirigidos a desarrollar el potencial de un solo o de ambos hemisferios cerebrales?; ¿Se logra algún cambio durante los estudios de educación superior, en la práctica los jóvenes universitarios egresan con la misma dominancia cerebral con que ingresaron o sucedieron cambios?

En la actualidad los estudiantes de psicología, al igual que el resto de los estudiantes universitarios, y sobre todo los de las carreras humanistas y de la salud, requieren desde los primeros años de estudio un proceso de autoconocimiento, de toma de conciencia individual y colectiva que les permita asumir el estudio de su carrera con mayor responsabilidad y compromiso. Es así como desde hace un tiempo, en el 2009, iniciamos la actividad de fortalecer en los estudiantes el descubrimiento de sus preferencias cerebrales, utilizando el cuestionario de Herrmann (1989), a fin de lograr una mayor comprensión de sus fortalezas y debilidades en el aprendizaje.

\section{Metodología}

De acuerdo con los objetivos de la investigación se adoptó los métodos descriptivo, en tanto se especifican las características del perfil de dominancia cerebral de los estudiantes de segundo año de psicología en distintos períodos académicos, y comparativo porque contrasta las cualidades relacionadas con los cuatro cuadrantes y los dos hemisferios cerebrales de los mismos.

El trabajo se realizó mediante la recolección y procesamiento de información, con la aplicación del instrumento denominado Cuestionario integral sobre mis Preferencias cerebrales, con el cual se determina los perfiles de estilos cerebrales de procesamiento de información o estilos de pensamiento, como lo designa Herrmann (1989). El instrumento fue aplicado a 75 ( $n=75)$ jóvenes estudiantes de la carrera de psicología en varios años, en la Universidad Nacional Autónoma de Nicaragua.

El cuestionario consta de 22 ítems que exploran diversos aspectos de la actividad de estudio y de la vida cotidiana. En cada uno de los ítems se presentan cuatro proposiciones que representa las distintas características comportamentales, procesales y de competencias de cada uno de los cuadrantes cerebrales. Luego de escoger la opción que más se acerca a la situación personal, se trasladan las respuestas a una plantilla de corrección. La evaluación consiste en determinar el grado de preferencia que se le asigna a cada cuadrante. Una vez hecho los cómputos necesarios en cada cuadrante y para determinar el tipo de utilización de los cuadrantes, se le asigna los siguientes códigos: Código 1; preferencia o dominancia primaria (más de 67 puntos). Código 2; Preferencia o dominancia secundaria o indecisión (entre 34 y 67 puntos). Código 3; Preferencia o dominancia terciaria o Rechazo no Preferencia (menos de 34 puntos). Es de hacer notar que el código 1 representa la verdadera preferencia o dominancia. Estas puntaciones 
pueden representarse gráficamente, trazando un círculo y dibujando un cuadrilátero resultante de las respuestas obtenidas.

Los perfiles de cuadrantes se representan por los números 1, 2 y 3 según los resultados obtenidos por cada uno, en la secuencia CI, LI, LD y CD. De acuerdo con lo anterior, el perfil 1-2-3-2, por ejemplo, significa que hay preferencia en el $\mathrm{Cl}$ (Cortical Izquierdo), preferencia secundaria en el LI (Límbico Izquierdo), preferencia terciaria en el LD (Límbico Derecho) y preferencia secundaria en el CD (Cortical Derecho).

Un perfil 1-1-1-1, significa que tiene preferencia primaria en todos los cuadrantes (cuádruple dominancia); en el extremo opuesto, el perfil 3-3-3-3 (cuádruple rechazo) y el perfil 2-2-2-2 significa que no hay dominancia ni rechazo en ninguno de los cuadrantes.

De tal manera que bajo todas las combinaciones posibles, pueden darse los grados de preferencia o dominancia nula, simple, doble, triple y cuádruple.

Tabla 1:

Perfiles de dominancia cerebral por cuadrante de toda la muestra.

\begin{tabular}{|l|c|c|}
\hline $\begin{array}{l}\text { Perfiles de dominancia } \\
\text { cerebral por cuadrantes } \\
\text { cerebrales }\end{array}$ & $\begin{array}{c}\text { Frecuencia absoluta } \\
\mathbf{n = 7 5}\end{array}$ & Frecuencia relativa \\
\hline Cortical izquierdo (ci) & 31 & $\mathbf{5 4 . 4 \%}$ \\
\hline Límbico izquierdo (li) & 24 & $42.0 \%$ \\
\hline Límbico derecho (ld) & 8 & $14.0 \%$ \\
\hline Cortical derecho (cd) & 12 & $21.0 \%$ \\
\hline
\end{tabular}

Fuente: Elaboración propia.

Los datos de la Tabla 1 evidencian que existe dominancia cerebral izquierda, tanto cortical como límbica, pues en ambos casos las cifras de sujetos clasificados de esta forma superan el $40 \%$. En contraste, las cifras de los que tienen dominancia derecha, en uno de los cuadrantes, solo alcanza, como máximo, una quinta parte de la muestra estudiada (14.0 y $21.0 \%$ ).

Tabla 2:

\section{Tipo de dominancia cerebral de toda la muestra}

\begin{tabular}{|l|c|c|}
\hline Tipos de dominancia & $\begin{array}{c}\text { Frecuencia absoluta } \\
\mathbf{n = 7 5}\end{array}$ & Frecuencia relativa \\
\hline Dominancia simple & 33 & $\mathbf{5 8 . 0 \%}$ \\
\hline Dominancia doble & 21 & $37.0 \%$ \\
\hline Sin dominancia & 4 & $7.01 \%$ \\
\hline Total & 57 & $100 \%$ \\
\hline
\end{tabular}

Fuente: Elaboración propia.

Los resultados de la Tabla 2 indican, ante todo, un predominio de la dominancia simple, la cual corresponde a más de la mitad de la muestra estudiada. El segundo lugar recae en los que tienen dominancia doble, en la cual fue clasificada poco más de la tercera parte de la misma (37.0\%) representado por 21 sujetos de la muestra. 
Por otro lado, destaca el hecho de que no existe dominancia triple ni cuádruple, lo cual supone que porcentajes importante de los sujetos solo utiliza una cuarta parte o la mitad del cerebro.

Tabla 3:

\section{Perfiles de doble dominancia cerebral de toda la muestra}

\begin{tabular}{|l|c|c|}
\hline $\begin{array}{l}\text { Perfiles de doble } \\
\text { dominancia cerebral }\end{array}$ & Frecuencia absoluta & Frecuencia relativa \\
\hline Hemisferio izquierdo (ci.li) & 13 & $62.0 \%$ \\
\hline Hemisferio derecho (ld-cd) & 1 & $4.76 \%$ \\
\hline Corticales (ci.cd) & 2 & $9.52 \%$ \\
\hline Límbicos (ld-li) & 2 & $9.52 \%$ \\
\hline Contralaterales (ci-ld) & 2 & $9.52 \%$ \\
\hline Contralatera (li-cd) & 1 & $4.76 \%$ \\
\hline Total & 21 & $100 \%$ \\
\hline
\end{tabular}

Fuente: Elaboración propia.

En la Tabla 3 en la que se analiza el $37 \%$ (21 sujetos) de la muestra que, según el instrumento posea doble dominancia, se logra apreciar que estos resultados constatan la casi exclusividad de la dominancia del hemisferio Izquierdo en detrimento del Derecho (62.0\% frente a $4.8 \%$ ), lo mismo que el bajo porcentaje del resto de clasificaciones (4.8 y $9.5 \%)$.

Estos resultados reflejan la dominancia del Cortical Izquierdo en todos los períodos (56.3, 63.2 y $46.0 \%$ ) y la dominancia del Límbico Izquierdo, aunque en menor porcentaje (47.4 y $40.9 \%$ ) en los dos últimos. Se destaca en el último período (2013), el aumento en la dominancia del Cortical derecho con un $40.9 \%$ y la doble dominancia únicamente en el 2010 con el $32.0 \%$, en el hemisferio izquierdo. También se enfatiza la poca dominancia del Límbico Derecho, tan importante para el profesional de la psicología.

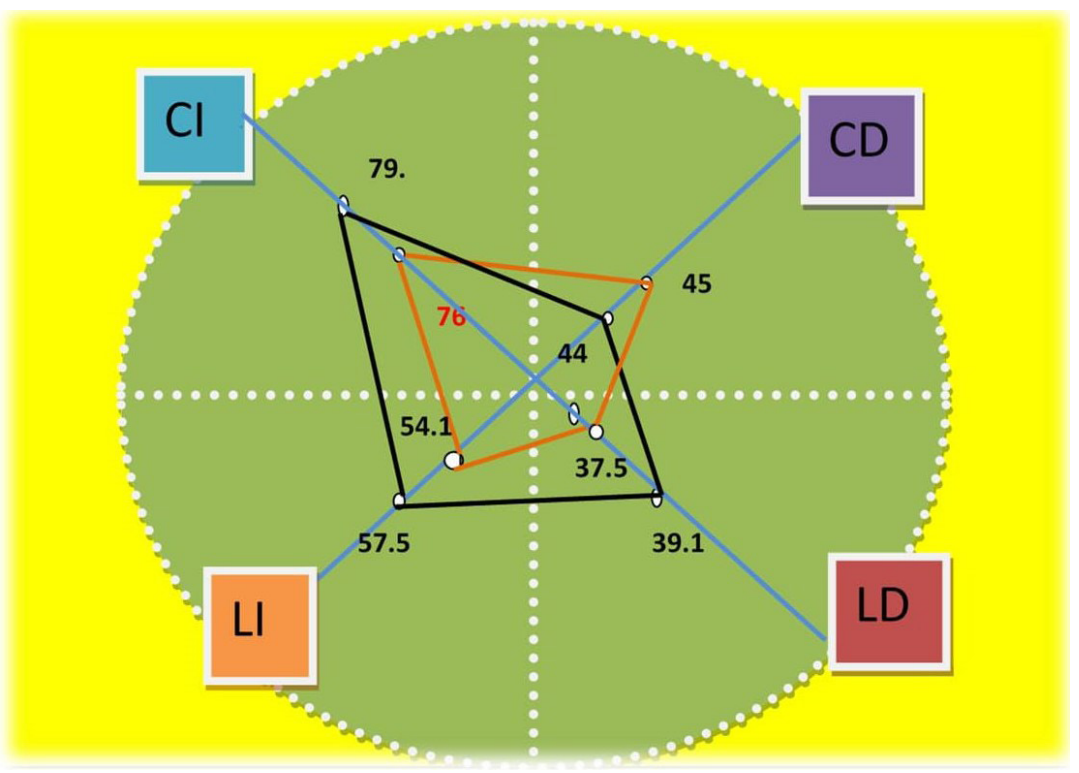

Gráfico 1. Dominancia cerebral por cuadrantes con base en el pre y post test. 
En el Gráfico 1 se muestra la dominancia cerebral por cuadrantes con base en el pre y post test (al inicio y final de la carrera) de la muestra de estudiantes escogida para tal efecto.

En este gráfico la figura externa está marcada en línea negra, y la figura interna, en línea naranja. Se observa que tanto en el pre y post test, se mantuvo la dominancia en el Cortical Izquierdo, aunque con una disminución en la puntuación media (79 y 76). De igual manera hubo disminución en la puntuación media del Límbico Izquierdo (57.5 - 54.1), y del Límbico Derecho con (39.137.5). Hay un aumento en el Cortical Derecho (44 en pre test y 45 en el post test).

\section{Discusión}

Al tener en cuenta las características de cada cuadrante y relacionarlas con los resultados de las tablas 1,3 y 4, se asume que el perfil general es consistente con los hallazgos de otros estudios, según los cuales la dominancia cerebral es mayoritaria para el cuadrante Cortical Izquierdo, seguido del Límbico Izquierdo.

Siguiendo a Herrmann (1995) y a Gardié (1997), puede afirmarse que la muestra estudiada está constituida por individuos que tienen facilidad para resolver problemas, dan importancia a la argumentación y a la crítica sobre la experiencia personal, a los hechos sobre la intuición, son fríos y arrogantes; además sus soluciones, aunque lógicas, resultan demasiado lineales y pocos creativas, les falta imaginación, creatividad, aptitudes para el arte y son demasiado individualistas. Sin embargo, desde la educación básica, la formación científica y la capacitación profesional las estrategias de procesamiento de información del cortical izquierdo deben estar presentes en el perfil deseable para cualquier población estudiantil.

Tabla 4:

Perfiles de dominancia cerebral por cuadrante y hemisferios.

\begin{tabular}{|l|c|c|c|c|c|c|}
\hline & \multicolumn{2}{c}{2009} & \multicolumn{2}{c}{2010} & \multicolumn{2}{c|}{2013} \\
\hline Cuadrantes y hemisferios & F & $\%$ & F & $\%$ & F & $\%$ \\
\hline Límbico Izquierdo (LI) & 9 & 56.3 & 12 & 63.2 & 10 & 46.0 \\
\hline Límbico Derecho (LD) & 6 & 31.3 & 9 & 47.4 & 9 & 40.9 \\
\hline Cortical Derecho (CD) & 2 & 12.5 & 3 & 16.0 & 4 & 48.2 \\
\hline Hemisferio Izquierdo (Ci-LI) & 0 & 0.0 & 3 & 16.0 & 9 & 40.9 \\
\hline Hemisferio Derecho (LD-CD) & 3 & 19.0 & 6 & 32.0 & 4 & 18.2 \\
\hline Contralaterales (CI-LD y LI-CD) & 0 & 0.0 & 1 & 5.3 & 1 & 4.5 \\
\hline
\end{tabular}

Fuente: Elaboración propia.

El límbico izquierdo, que es otro de los cuadrantes con mayor dominancia en la muestra y conformado por estrategias operacionales dirigidas fundamentalmente a la ejecución de acciones, previamente diseñadas, podría aseverarse que en él no hay espacio para la reflexión crítica ni para la búsqueda de alternativas distintas a las establecidas por las normas de la tradición o planificación. En resumen, no ofrece demasiada flexibilidad, únicamente se pone en marcha de manera controlada, secuencial y previsible. Es un incansable trabajador.

En estas mismas tablas se manifiesta la pobreza de dominancia del Límbico Derecho, cuadrante necesario a desarrollar y a utilizar con mayor frecuencia, en tanto son estudiantes de psicología. El Límbico es eminentemente comunicativo y expresivo, empático. Representa una visión más hacia afuera que hacia adentro del individuo, con clara tendencia a involucrarse en relaciones interpersonales de cooperación y amistad, y en proyectos comunitarios de asistencia y orientación de salud social 
En la Tabla 2 se observa que los estudiantes presentan en mayor medida una dominancia simple (58\%), es decir dominancia primaria en un cuadrante, seguida de una dominancia doble (37\%). Esta distribución permite identificar el patrón educacional de la población estudiantil. De acuerdo con Chalvin (2003, p. 82), las personas que han desarrollado dominancia simple se caracterizan por tener menos conflictos internos y tomar decisiones más coherentes y previsibles. La capacidad para interactuar es reducida, al igual que su capacidad de independencia creativa. Lo anterior es aplicable a la doble dominancia en un mismo hemisferio con la salvedad que en ellos se amplía la calidad del pensamiento.

En la Tabla 4 se resalta el incremento de dominancia del Cortical Derecho en el 2013 (40.9\%). Este cuadrante presenta dos características especiales. Tradicionalmente se le ha subvalorado y algunos lo consideran el "cuadrante de la creatividad" en tanto es original, espacial, simultáneo, tiene la capacidad de generar situaciones novedosas, no convencionales, realizadas y planeadas con anticipación, de forma consciente por medio de la imaginación. Chalvin le denomina el "Estratega". En la muestra estudiada de manera persistente, en los diferentes períodos, es el de menor preferencia. Se podría decir que el estilo del pensamiento del cuadrante cortical derecho presenta escasa preferencia, situación de desventaja para los estudiantes en general.

En el Gráfico 1, llama la atención que los resultados del pre y post test son similares, una dominancia del hemisferio izquierdo, en detrimento del hemisferio derecho, sobre todo el cuadrante cortical izquierdo con puntuaciones arriba de la media (79 y 76). También es pertinente observar que en tres de los cuadrantes (Cl, LI y LD) disminuyeron en el post test. Por último y no menos importante, a pesar de que en el CD se observa aumento de un punto en el post test de 44 a 45, no hay dominancia en él.

\section{Conclusiones}

Los resultados observados confirman lo que otros estudios han encontrado: la dominancia del hemisferio izquierdo y sobre todo del cortical izquierdo sobre los otros cuadrantes. La dominancia simple que implica la subutilización del cerebro como totalidad. El desaprovechamiento del hemisferio derecho. En el caso del pre y post test, se puede decir que el paso por la educación superior no implica desarrollar nuevas preferencias cerebrales que las que ya traen al ingresar a ella.

La implicancia de estos resultados en la educación radica en la necesidad de no solo adecuar los métodos y técnicas de enseñanza para entrar en sintonía con el perfil de la mayoría de los estudiantes (al no encontrar alguna diferencia estadísticamente significativa en las puntuaciones de pre y post test, entonces el cuerpo estudiantil se beneficiaría de métodos de aprendizaje orientado a personas con una dominancia cerebral del hemisferio Izquierdo), sino también que las actividades académicas sean dirigidas a la potencialización y utilización de los dos hemisferios, para de esta manera contribuir a una mayor utilización del cerebro en su totalidad.

La universidad podría beneficiarse tremendamente al institucionalizar este tipo de diagnóstico, para así crear un mapa de las habilidades de sus estudiantes basadas en la teoría del cerebro total de Herrmann. De esta manera se podría diseñar contenido teórico y práctico orientado específicamente al estilo predominante de una carrera o salón de clases. Entre otros, permitiría teóricamente un mejor aprovechamiento de la educación superior y una disminución de los abandonos. Un análisis detallado del tipo de dominancia cerebral predominante en un salón de clases y la subsecuente utilización de metodologías orientadas para dicha predominancia convertiría la UNAN en una pionera en la utilización de la neurociencia aplicada al aprendizaje de profesionales, facilitando una educación muchísimo más eficiente, eficaz y satisfactoria. La universidad enseñaría más y mejor, produciría mejores resultados en sus estudiantes. 
En esta misma línea de análisis, la presente investigación confirma que no existe un cambio en la dominancia cerebral en comparación con el momento en que los estudiantes ingresan a la educación superior y el momento que finalizan. Esto constituye una clara crítica y una falla dentro del sistema educativo universitario. De institucionalizar este método de diagnóstico, cada departamento académico podría diseñar metodologías orientadas a potencializar la utilización de los cuadrantes cerebrales en cada una de las actividades académicas y en la estructuración de los grupos de trabajo del salón de clase. De esta manera, la universidad se convertiría en un verdadero formador no solo del área profesional/académica, sino también de una cualidad mucho más holística, aunando a la formación de ciudadanos profesionales con alto pensamiento crítico, enfocados a la resolución de problemas no convencionales, con agudeza creativa e innovadora y con franca capacidad de relacionarse con él y los otros de forma humana y comprometida.

\section{Referencias bibliográficas}

- Calle, M. G., Cleves, N. R., \& Burgos, B. M. (2008). Relación entre los perfiles de dominancia cerebral de los estudiantes de primero, quinto y décimo semestres delPrograma de Bacteriología y Laboratorio Clínico de la Universidad Colegio Mayor de Cundinamarca. Nova,6(9), 40. doi:10.22490/24629448.395

- Caza. P., (2005) Modelo de los cuadrantes generales (disponible en: http://www.rmm.cl/ index

- Chalvin. M. J., (2003): Los dos cerebros en el aula. (3a. ed.) Madrid: TEA Ediciones. Traducción: Ma. Victoria de la Cruz.

- Gómez. A. Recio. R. Gómez. D. Lóez. H. (2010): Diagnóstico de estilo de aprendizaje en estudiantes universitarios de nuevo ingreso basado en la dominancia cerebral. Revista Estilos de aprendizaje, num. 5, vol 5, 2010. (Disponible en http://www.uned.es/ revistaestilosdeaprendizaje/numero_5/articulos/lsr_5_articulo_4.pdf). Consultada en abril 2012.

- Kandel, E. R., Schwartz, J. H., \& Jessell, T. M. (2008). Neurociencia y conducta. Madrid: Pearson Educación.

- Martínez. M. (1992). El proceso creador a la luz de la neurociencia. Publicación: Comportamiento 1993. V2 n1. (Disponible en: http://www.comportamiento.dsm.usb.ve/ revista/vol2_n1_93.pdf

- Publication manual of the American Psychological Association. (2016). Brantford, Ontario: W. Ross MacDonald School Resource Services Library.

- Rómulo. O, Marval. O., (2000): Avances de las neurociencias. Implicaciones en la educación. Revista Agenda académica 2007. v7, n2. (Disponible en: http://www.virtual.unal.edu.co/ cursos/humanas/mtria_edu/2021076/und_1/pdf/lectura3m1estacion2.pdf. Consultada en enero 2010).

- $\quad$ Rojas, G., Salas, R., \& Jimenez, C. (2006). Estilos De Aprendizaje Y Estilos De Pensamiento Entre Estudiantes Universitarios. Estudios Pedagógicos (Valdivia),32(1). doi:10.4067/s071807052006000100004 